\title{
Dynamics of two-sign point vortices in positive and negative temperature states
}

\section{$\operatorname{AUTHOR}(\mathrm{S}):$}

Yatsuyanagi, Y; Kiwamoto, Y; Tomita, H; Sano, MM; Yoshida, T; Ebisuzaki, T

\section{CITATION:}

Yatsuyanagi, Y ...[et al]. Dynamics of two-sign point vortices in positive and negative temperature states. Physical Review Letters 2005, 94(5): 054502.

\section{ISSUE DATE:}

2005-02-11

URL:

http://hdl.handle.net/2433/50244

RIGHT:

Copyright 2005 American Physical Society 


\title{
Dynamics of Two-Sign Point Vortices in Positive and Negative Temperature States
}

\author{
Yuichi Yatsuyanagi, ${ }^{*}$ Yasuhito Kiwamoto, Hiroyuki Tomita, Mitsusada M. Sano, and Takeshi Yoshida \\ Graduate School of Human and Environmental Studies, Kyoto University, Sakyo, Kyoto 606-8501, Japan
}

Toshikazu Ebisuzaki

RIKEN (The Institute of Physical and Chemical Research), Wako, Saitama 351-0198, Japan

(Received 5 August 2004; published 7 February 2005)

\begin{abstract}
Dynamics of two-sign point vortices in two-dimensional circular boundary is examined by numerical simulations with MDGRAPE-2. The vortex system is characterized by the inverse temperature $\beta$ as determined from the density of states of the microcanonical ensemble of numerically generated $10^{7}$ states. The massive simulation shows that different configurations appear in the time-asymptotic state depending on the sign of $\beta$. Condensation of the same-sign vortices is observed when $\beta<0$, while the both-sign vortices tend to be uniformly neutralized when $\beta>0$. During the condensation, a part of the vortices gains energy to form clumps (patches), and the other part of the vortices loses energy to keep the total energy constant and mixes with vortices of the other sign. This observation demonstrates a characteristic feature of negative $\beta$ states that the system energy concentrates into the clumps of the same-sign vortices.

PACS numbers: 47.32.Cc, 05.10.-a, 47.11.+j
\end{abstract}

Recent studies of vortices have received continuing impetus from a broad variety of problems arising in physics, for example, coherent structure in turbulent flows in hydrodynamics [1] and magnetohydrodynamics [2], vortex patterns in superfluid ${ }^{4} \mathrm{He}$ and Bose-Einstein condensates [3], magnetic vortices in superconducting materials [4], and Kosterlitz-Thouless transition [5]. The thread of modern interest in a two-dimensional (2D) point vortex can be traced back to Kirchhoff [6], and there still remain many issues, including stability properties and crystallization of $\mathrm{N}$-vortex patches [7-9], and the inverse cascade problem of energy [10]. Some of these problems have been tackled experimentally using non-neutral plasmas [11-15]. The macroscopic dynamics of the non-neutral plasma is identical with 2D incompressible ideal fluid that obeys the Euler equation. A remarkable feature of such fluid is a negative temperature state suggested by Onsager [16].

In this Letter, we numerically examine the dynamics of two-sign point vortices in positive and negative temperature states contained in a cylinder of radius $R$ using the fast calculating special-purpose computer for molecular dynamics simulations, MDGRAPE-2 [17,18]. The system is characterized by the inverse temperature $\beta$ that is statistically determined from the entropy $S=\ln W(E, I)$ as

$$
\beta \equiv \frac{1}{T}=\frac{d S}{d E}=\frac{1}{W(E, I)} \frac{d W(E, I)}{d E},
$$

where the Boltzmann constant is normalized to unity. The density of states $W(E, I)$ is evaluated as a function of the system energy $E$ and the angular impulse $I$ by employing the large-scale numerical sampling $\left(10^{7}\right.$ times $)$ for a microcanonical ensemble of a system consisting of $N / 2$ positive and $N / 2$ negative point vortices (typically $N=6724$ ). Compared to the previous numerical evaluations of the density of states for systems with $N \geq 40$ [19-21], the number of sampling is larger at least by a factor of $10^{2}$, so that we can discuss the asymptotic form of $W(E, I)$ over a wider range of $E$. To obtain time-asymptotic behavior of the vortex distribution, we trace the time evolution of the system from various initial distributions using MDGRAPE-2. The condensation of the same-sign vortices was demonstrated by the numerical simulations under a limited system scale or the mean field approximation $[22,23]$. However, MDGRAPE-2 breaks such limitations. The velocity field is exactly evaluated at the position of the particle, so that the massive time-development simulations provide the time-asymptotic equilibrium distributions that may be comparable in spatial resolution to the static equilibria discussed in Refs. [22-25]. The main features observed are (a) the clear demonstration that the same-sign vortices condense when $\beta<0$ but opposite sign vortices mix up when $\beta>0$, and (b) the finding that, when $\beta<0$, the energy accumulates in the clumps of the same-sign vortices while vortices excluded from the clumps are mixed in the background to share a smaller amount of energy.

Let us consider a system consisting of $N / 2$ point vortices with the circulation of $\Gamma_{0}(>0)$ and the same number of vortices with $-\Gamma_{0}$. Because $N$ image vortices are induced by the presence of the circular boundary of radius $R$, the Hamiltonian of the system is given by [26]

$$
\begin{aligned}
H= & -\frac{1}{4 \pi} \sum_{i}^{N} \sum_{j \neq i}^{N} \Gamma_{i} \Gamma_{j} \ln \left|\boldsymbol{r}_{i}-\boldsymbol{r}_{j}\right|+\frac{1}{4 \pi} \sum_{i}^{N} \sum_{j}^{N} \Gamma_{i} \Gamma_{j} \ln \left|\boldsymbol{r}_{i}-\overline{\boldsymbol{r}}_{j}\right| \\
& -\frac{1}{4 \pi} \sum_{i}^{N} \sum_{j}^{N} \Gamma_{i} \Gamma_{j} \ln \frac{R}{\left|\boldsymbol{r}_{j}\right|},
\end{aligned}
$$

where $\boldsymbol{r}_{i} \equiv\left(x_{i}, y_{i}\right)$ and $\Gamma_{i}$ are the position vector and the circulation of the $i$ th point vortex, respectively. The image vortex of the $i$ th vortex is located at $\overline{\boldsymbol{r}}_{i}=R^{2} \boldsymbol{r}_{i} /\left|\boldsymbol{r}_{i}\right|^{2}$. The 
last term in Eq. (2) is introduced so that the value of the stream function on the circular boundary is fixed at zero. Note that the system has an additional constant of motion, i.e., an angular impulse of the point vortices $I=\sum_{i}^{N} \Gamma_{i}\left|\boldsymbol{r}_{i}\right|^{2}$.

Since the conjugate variables of the system are $\left(x_{i}, y_{i}\right)$, the system has only a finite amount of total phase space volume available, $\left(\pi R^{2}\right)^{N}$. Thus, the density of states $W(E, I)$ is expected to converge to zero in the limit of $E \rightarrow \infty$. To obtain $W(E, I)$ explicitly, we accumulate the states for the microcanonical ensemble of the vortex system, which are generated by randomly distributing positive and negative point vortices within the boundary $[19,21]$. For each sampled state, we calculate the energy $E$ and the angular impulse $I$. The state is then binned into the cell bounded by $E$ and $E+d E$ and by $I$ and $I+d I$. Figure 1 summarizes the numerical results obtained after the sampling of $10^{7}$ states for the $N=6724$ vortex system by plotting $W(E, I) d E d I$ at $I=0$. We observe that $W(E, 0) d E d I$ has a maximum at $E=29.0\left(\equiv E_{c}\right)$ as expected.

The validity of the number of sampling is examined in Fig. 2. Here we plot three cardinal parameters, i.e., $E_{c}(\bigcirc)$, the upper and the lower half widths ( $\square$ and $\triangle$ ) at half maximum of $W(E, 0) d E d I$, for the different numbers of sampling. The difference in the upper and the lower half widths shows that the profile of $W(E, 0) d E d I$ is asymmetric about $E=E_{c}$. Because these parameters remain almost constant beyond the sampling of $6 \times 10^{6}$ states, the profile in Fig. 1 may be guaranteed to correctly represent the density of states for the $N=6724$ vortex system. Additional numerical analyses indicate that the profile of $W(E, 0) d E d I$ depends on the number of point vortices $N$. As shown in Fig. 3, the critical energy $E_{c}$ and the full width at half maximum decrease in proportion to $N^{-1}$ with the total circulation kept constant. The observed scaling predicts that $E_{c} \rightarrow 0$ as $N \rightarrow \infty$ (i.e., continuous fluid), and the profile of $W(E, 0)$ approaches the Dirac delta function.

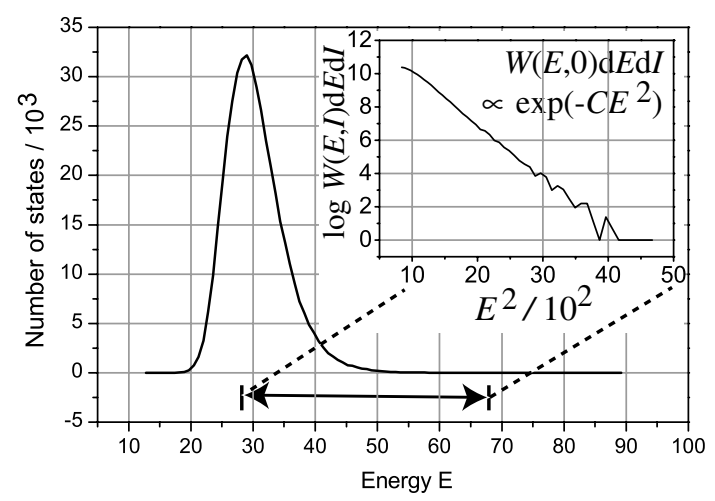

FIG. 1. Number of states $W(E, I) d E d I$ at $I=0$ is plotted. The peak value is at $E=29.0\left(=E_{c}\right)$. Inset shows that $W(E, 0) d E d I$ is proportional to $\exp \left(-C E^{2}\right)$ at $E>E_{c}$.
The inverse temperature $\beta$ is determined from the distribution shown in Fig. 1 by using Eq. (1). Apparently, the temperature is negative (positive) for the system with energy $E>E_{c}\left(E<E_{c}\right)$. For the system of $E>E_{c}$, as indicated by the inset, the density of states scales as $W(E, 0) d E d I \propto \exp \left(-C E^{2}\right)$, and we obtain the relation $[19,21]$

$$
\beta=\frac{\partial \log W(E, 0)}{\partial E} \propto-2 C E,
$$

where $C$ is a constant. We should note that, for $E>E_{c}$, $\beta(<0)$ goes down with the increase of $E$.

In the next, we demonstrate the time-asymptotic vortex distributions obtained by the time-development simulations. Two results are shown in Figs. 4 and 5. The equation of motion of the point vortices is derived from Eq. (2) as [23]

$$
\frac{d \boldsymbol{r}_{i}}{d t}=-\frac{1}{2 \pi} \sum_{j \neq i}^{N} \Gamma_{j} \frac{\left(\boldsymbol{r}_{i}-\boldsymbol{r}_{j}\right)}{\left|\boldsymbol{r}_{i}-\boldsymbol{r}_{j}\right|^{2}}+\frac{1}{2 \pi} \sum_{j}^{N} \Gamma_{j} \frac{\left(\boldsymbol{r}_{i}-\overline{\boldsymbol{r}}_{j}\right)}{\left|\boldsymbol{r}_{i}-\overline{\boldsymbol{r}}_{j}\right|^{2}} .
$$

To accelerate the calculation of the Biot-Savart integrals on the right-hand side of Eq. (4), we use MDGRAPE-2 $[27,28]$. In the initial distribution, the centers of the clumps are arranged on the circle of radius $R_{c}$ at regular angular intervals. Each clump consists of the same-sign vortices, and the polarity of the clump changes in turn in a clockwise direction. The number of vortices $N$ is chosen at the same order as the one used for Fig. 1. The control parameters are the number of clumps $n_{c}$, the initial radius of each clump $r_{c}$, and the time scale of self-rotation of a clump in unbounded plane $\tau_{c}$. In Fig. 4, the initial conditions are $E=2.69 \times 10^{4}\left(\equiv E_{1}>E_{c}\right)$ and $I=2.28 \times 10^{-9} \approx 0$ so that the temperature is negative. At $\tau=32$ the polarity of the upper right clump is positive and that of the lower left clump is negative. We can see that the vortices tend to form the clumps of the same sign [22]. This phenomenon represents the vortex condensation. It may correspond to the inverse cascade that is an energy transfer process from

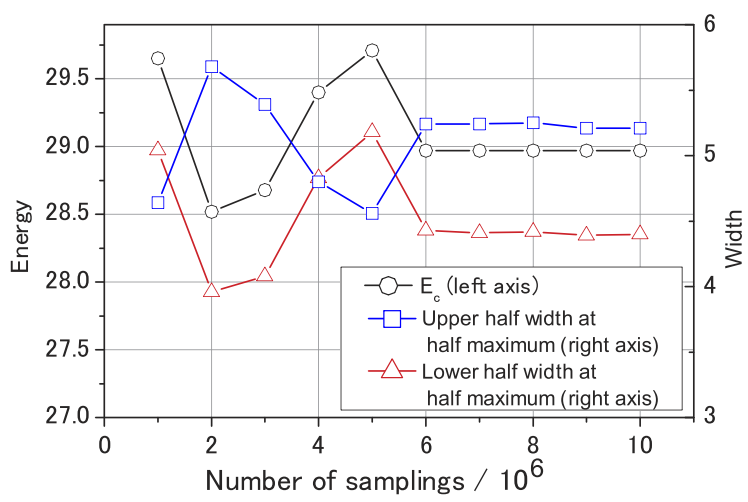

FIG. 2 (color online). The critical energy $E_{c}$ and the upper and the lower half widths at half maximum of $W(E, 0) d E d I$ are plotted against the number of samplings. 


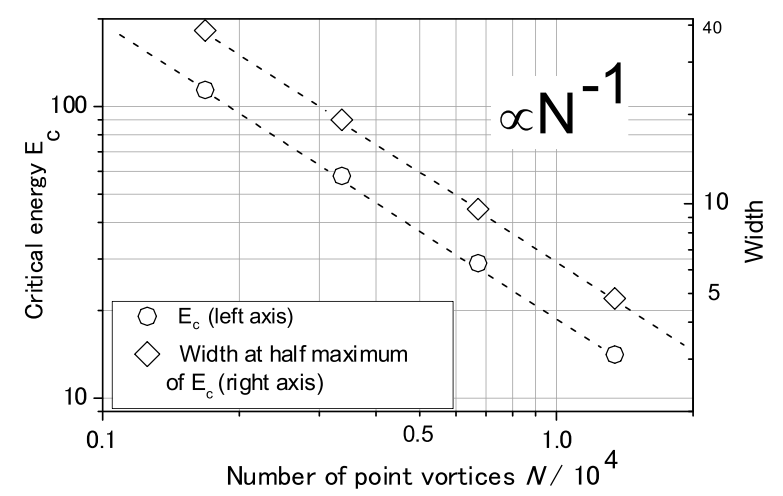

FIG. 3. Critical energy $E_{c}$ and width at half maximum are plotted as a function of $N$. In each case, the number of sampling is $10^{7}$.

a small scale to a large scale [16]. In Fig. 5, the initial conditions are $E=24.1\left(\equiv E_{2}<E_{c}\right)$ and $I_{1}=-1.17 \times$ $10^{-13} \approx 0$ so that the temperature is positive. The vortices diffuse over the circular cross section, and the positive and the negative vortices mix almost uniformly. This is a common feature observed in systems with positive temperature and corresponds to a normal cascade.

Snapshots of the asymptotic states corresponding to different values of energy are shown in Fig. 6. The inverse temperatures $\beta_{2}, \beta_{3}$, and $\beta_{4}$ associated with the system energy $E_{2}, E_{3}$, and $E_{4}$, respectively, satisfy $\beta_{2}>0>\beta_{3}>$ $\beta_{4}$. The small clumps (spots) are created as a result of the vortex condensation in the negative temperature cases in Figs. 6(b) and 6(c). It is noticeable that the size of the clump depends on the temperature. The size becomes larger as the inverse temperature $\beta$ goes down at $\beta<0$.

The other essential feature of the negative temperature system is the energy concentration in a specific group of the vortices. There is no viscous term in the equation of motion (4), and the reduction of the total energy due to the numerical dissipation is evaluated to be less than $0.1 \%$ in Fig. 4. Thus, the total energy is conserved in the simulation. If the energy of a part of the system increases, the energy of the rest should decrease. In Fig. 4, "a part of the system" is the vortices inside the clumps, and the rest is the background vortices (outside the clumps). We evaluate the time evolution of the energies belonging to the clumps and the background vortices for the case in Fig. 4. The result is shown in Fig. 7. The energy of the vortices that constitutes

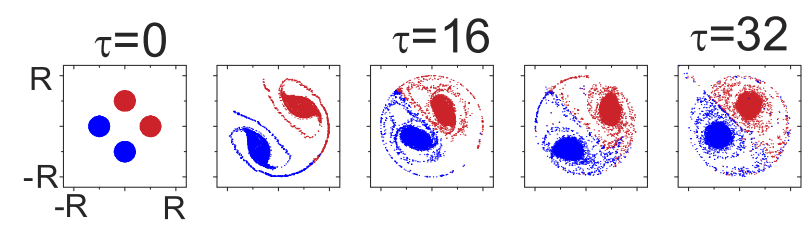

FIG. 4 (color online). Time evolution of the negative temperature system is plotted. The values of the parameters are $N=$ 6724, $n_{c}=4, R_{c}=0.5 R, r_{c}=0.2 R, \tau_{c}=15.6$, and $E=$ $2.69 \times 10^{4}\left(=E_{1}\right)$.
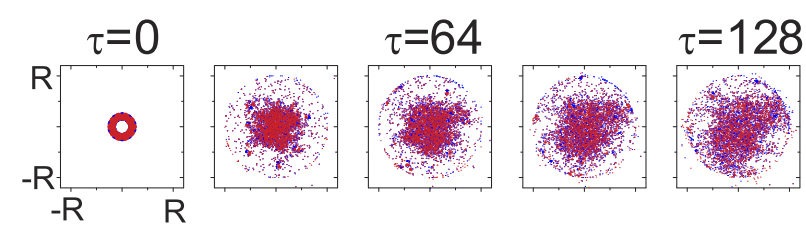

FIG. 5 (color online). Time evolution of the positive temperature system is plotted. The values of the parameters are $N=$ 6920, $n_{c}=20, R_{c}=0.2 R, r_{c}=0.07 R, \tau_{c}=9.3$, and $E=$ $24.1\left(=E_{2}\right)$. Because the clumps overlap each other, we see only the outline of the clumps (hollow ring) at $\tau=0$.

the clumps certainly increases during the initial period up to $\tau=20$ when the formation of almost circular clumps is finished. On the other hand, the energy belonging to the background vortices goes down. The time traces of the energies belonging to the two groups are symmetric about the line representing the ratio of 0.5 . After $\tau=20$, the energy of each group remains constant. It is noticeable that the exchange of the energy between the two groups of the vortices enables the vortex condensation. This observation indicates the common and essential role of background vortices in supporting the condensation of two-sign vortices as well as in assisting the generation of symmetric configuration of non-neutral plasma clumps [9,12-15].

Let us briefly examine the mechanism to determine the asymptotic configuration of the two clumps by employing a simple model. Suppose that the clumps are confined uniformly within two circular patches of radius $0.2 R$ that are separated by the distance of $2 r$ across the center of the circular boundary of radius $R$. A calculation indicates that the energy of the clump system is maximized for $r \approx 0.5 R$. This maximum-energy configuration has a noticeable similarity to the distribution obtained at $\tau=32$ in Fig. 4. Though the contribution of the background vortices is neglected by assuming they are sufficiently neutralized, the model consideration suggests that the clumps tend to share the maximum energy available under the constraint of conservation of the system energy and the angular impulse. It is consistent with the common feature of the negative temperature system.

In summary, we have numerically examined the dynamics of two-sign point vortices confined in the circular boundary. The state of the vortex system is characterized
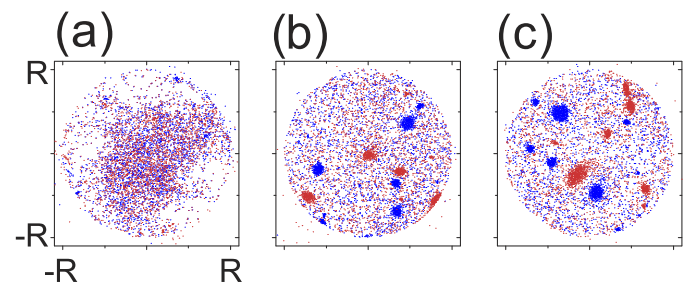

FIG. 6 (color online). Snapshots of different energies (a) $E_{2}=24.1$, (b) $E_{3}=142$, and (c) $E_{4}=315$ at $\tau=128$ are shown. 


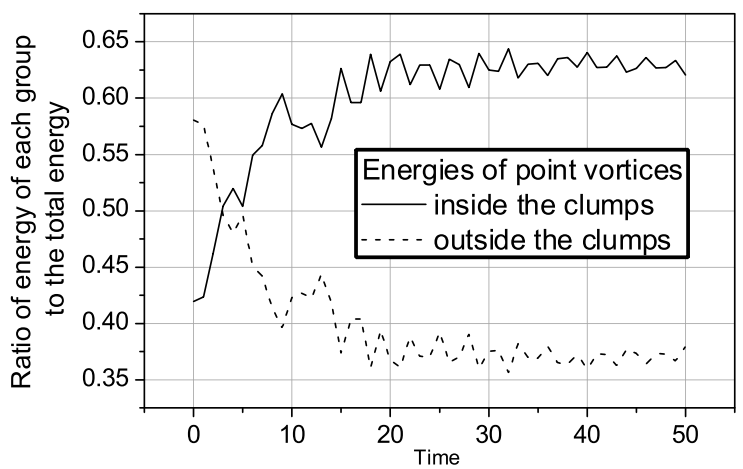

FIG. 7. Time evolution of the vortex energies inside and outside the clumps. We define a clump as a circular area of radius $0.2 R$ that coincides with the initial radius of the clump. The grouping in each time step is inapplicable during $\tau=0$ through 4 , because the transition from four to two clumps is not completed yet in this period and there is no clumplike distribution. Thus, we use the grouping data obtained at $\tau=4$ for the energy calculations between $\tau=0$ and 4 .

by the temperature that is determined from the density of states. By using the large-scale numerical sampling for the microcanonical ensemble, we determine the density of states. The time-asymptotic vortex distribution of the system with given energy and angular impulse shows a clear correlation with the temperature. At negative temperature, the same-sign vortices tend to condense into clumps by gaining the energy from other vortices remaining in the background. The correspondence between the vortex dynamics and the temperature supports the long-standing interpretation in terms of the negative temperature. Remaining issues include the precise evaluation of the density of states at a high system energy $E>60$, in-depth physical interpretation of $E_{c} \propto N^{-1}$ at negative temperature, and morphology of the clumps in the time-asymptotic states.

This work was supported by the Japan Society for the Promotion of Science for Young Scientists.

*Electronic address: yyanagi@ phys.h.kyoto-u.ac.jp

[1] A. J. Chorin, Vorticity and Turbulence (Springer-Verlag, Berlin, 1994).
[2] D. Biskamp, Nonlinear Magnetohydrodynamics (Cambridge University, Cambridge, 1993).

[3] A. L. Fetter and A. A. Svidzinsky, J. Phys. Condens. Matter 13, R135 (2001).

[4] A. Tonomura, Nature (London) 397, 308 (1999).

[5] J. M. Kosterlitz and D. J. Thouless, J. Phys. C 6, 1181 (1973).

[6] G. R. Kirchhoff, Vorlesungen ueber Mathematische Physik (Teubner, Leipzig, 1883), Vol. I.

[7] L. J. Campbell Phys. Rev. A 24, 514 (1981).

[8] H. Aref, P. K. Newton, M. A. Stremler, T. Tokieda, and D. L. Vainchtein, Adv. Appl. Mech. 39, 1 (2003).

[9] T. Yoshida and M. M. Sano (to be published).

[10] R. Kinney, J.C. McWilliams, and T. Tajima, Phys. Plasmas 2, 3623 (1995).

[11] Y. Kiwamoto, K. Ito, A. Sanpei, and A. Mohri, Phys. Rev. Lett. 85, 3173 (2000).

[12] A. Sanpei, Y. Kiwamoto, K. Ito, and Y. Soga, Phys. Rev. E 68, 016404 (2003).

[13] Y. Soga, Y. Kiwamoto, A. Sanpei, and J. Aoki, Phys. Plasmas 10, 3922 (2003).

[14] D.Z. Jin and D.H.E. Dubin, Phys. Rev. Lett. 80, 4434 (1998).

[15] K. S. Fine, A.C. Cass, W. G. Flynn, and C.F. Driscoll, Phys. Rev. Lett. 75, 3277 (1995).

[16] L. Onsager, Nuovo Cimento Suppl. 6, 279 (1949).

[17] T. Narumi, R. Susukita, T. Ebisuzaki, G. McNiven, and B. Elmegreen, Mol. Simul. 21, 401 (1999).

[18] R. Susukita et al., Comput. Phys. Commun. 155, 115 (2003).

[19] C. E. Seyler, Jr., Phys. Fluids 19, 1336 (1976).

[20] D. J. Johnson, Phys. Fluids 31, 1856 (1988).

[21] O. Bühler, Phys. Fluids 14, 2139 (2002).

[22] G. Joyce and D. Montgomery, J. Plasma Phys. 10, 107 (1973).

[23] Y. B. Pointin and T. S. Lundgren, Phys. Fluids 19, 1459 (1976).

[24] S. Kida, J. Phys. Soc. Jpn. 39, 1395 (1975).

[25] R. A. Smith and T. M. O'Neil, Phys. Fluids B 2, 2961 (1990).

[26] P. K. Newton, The N-Vortex Problem (Springer-Verlag, Berlin, 2001), Chap. 3.

[27] Y. Yatsuyanagi, T. Ebisuzaki, T. Hatori, and T. Kato, Phys. Plasmas 10, 3181 (2003).

[28] Y. Yatsuyanagi, Y. Kiwamoto, T. Ebisuzaki, T. Hatori, and T. Kato, Phys. Plasmas 10, 3188 (2003). 